\title{
Spared Anterograde Memory for Shock-Probe Fear Conditioning After Inactivation of the Amygdala
}

\author{
Hugo Lehmann, ${ }^{1,4}$ Dallas Treit, ${ }^{2}$ and Marise B. Parent ${ }^{2,3}$ \\ ${ }^{1}$ Center for Studies in Behavioral Neurobiology, Concordia University, Montreal, Quebec, Canada H4B 1R6; ${ }^{2}$ Department of \\ Psychology, University of Alberta, Edmonton, Alberta, Canada T6G 2E9; ${ }^{3}$ Department of Psychology, Georgia State University, \\ Atlanta, Georgia 30303, USA
}

\begin{abstract}
Previous studies have shown that amygdala lesions impair avoidance of an electrified probe. This finding has been interpreted as indicating that amygdala lesions reduce fear. It is unclear, however, whether amygdala-lesioned rats learn that the probe is associated with shock. If the lesions prevent the formation of this association, then pretraining reversible inactivation of the amygdala should impair both acquisition and retention performance. To test this hypothesis, the amygdala was inactivated (tetrodotoxin; TTX; $1 \mathrm{ng} /$ side) before a shock-probe acquisition session, and retention was tested $4 \mathrm{~d}$ later. The data indicated that, compared with rats infused with vehicle, rats infused with TTX received more shocks during the acquisition session, but more importantly, were not impaired on the retention test. In Experiment 2, we assessed whether the spared memory on the retention test was caused by overtraining during acquisition. We used the same procedure as in Experiment 1, with the exception that the number of shocks the rats received during the acquisition session was limited to four. Again the data indicated that amygdala inactivation did not impair performance on the retention test. These results indicate that amygdala inactivation does not prevent the formation of an association between the shock and the probe and that shock-probe deficits during acquisition likely reflect the amygdala's involvement in other processes.
\end{abstract}

The ability to learn and remember the relationships between aversive events and the stimuli that predict them is an adaptive capacity essential to the survival of animals. Fear conditioning, a paradigm in which the subject learns that an initially neutral stimulus is associated with a fear-eliciting stimulus or event, is typically used to study the neural mechanisms of learned fear. Extensive evidence indicates that the amygdala is involved in the learning and memory of conditioned fear. For instance, amygdala lesions may induce anterograde and retrograde amnesia for instrumental and Pavlovian fear conditioning (Liang et al. 1982; Dunn and Everitt 1988; Phillips and LeDoux 1992; Sananes and Davis 1992; Kim and Davis 1993; Parent et al. 1995a; LaBar and LeDoux 1996; Lee et al. 1996; Maren et al. 1996; Bermudez-Rattoni et al. 1997; Muller et al. 1997; Maren 1998, 1999; Poremba and Gabriel 1999; Wilensky et al. 1999; Antoniadis and McDonald 2001).

In contrast, other evidence indicates that the amygdala is involved in, but not necessary for the retention of conditioned fear (Vanderwolf et al. 1988; Sutherland and McDonald 1990; Helmstetter 1992; Parent et al. 1992, 1994, 1995b; Helmstetter and Bellgowan 1994; Killcross et al. 1997; Cahill et al. 2000; Lehmann et al. 2000). For example, we recently demonstrated that the amygdala does not appear to be necessary for retrograde memory for cued fear conditioning (Lehmann et al. 2000). The findings showed that rats given amygdala lesions $4 \mathrm{~d}$ after being exposed to an electrified probe did not show any deficits when memory was tested after the induction of the lesions. Specifically, avoidance of the probe on the retention test did not differ between lesioned and control rats. Furthermore, retention was better in shock-experienced lesioned rats than in lesioned rats that had been exposed to a nonelectrified probe during training (i.e., shock-naive).

\section{${ }^{4}$ Corresponding author.}

E-MAIL hlehmann@vax2.concordia.ca; FAX (514) 848-4545.

Article and publication are at http://www.learnmem.org/cgi/doi/10.1101/ Im.54103.
Although shock-probe avoidance on the retention test was not affected in shock-experienced rats, it was impaired in shocknaive lesioned rats. On the retention test, amygdala-lesioned, shock-naive rats received more shocks and approached the probe more quickly than did control shock-naive rats. This replicates the findings of several studies showing that pretraining amygdala lesions impair shock-probe avoidance (Kopchia et al. 1992; Treit et al. 1993; Treit and Menard 1997). The retention test in shocknaive rats was essentially equivalent to examining the effects of pretraining amygdala lesions in this group because the retention test was the first time the shock-naive lesioned rats received shock.

One limitation encountered with lesions that induce permanent cell damage (i.e., neurotoxic or electrolytic) is that it is difficult to determine whether the behavioral deficits observed are caused by an impairment in learning, retention, and/or performance. Shock-probe avoidance deficits caused by pretraining permanent amygdala lesions may reflect an inability to form an association between the shock and the probe or may indicate a deficit in some other process, such as unconditioned fear. We hypothesized that if the lesions prevent the formation of this association, then pretraining reversible inactivation of the amygdala should impair both acquisition and retention performance. In the present study, the amygdala was inactivated immediately before a first shock-probe session (acquisition) and memory was assessed $4 \mathrm{~d}$ later in a second shock-probe session (retention).

Reversible lesions were used to avoid any lasting effects of the lesions on the retention test. We used the sodium channel blocker tetrodotoxin (TTX) to induce the reversible lesions because many studies investigating the neural correlates of learning and memory have shown that TTX is an effective method for temporarily inactivating a targeted structure (Bucherelli et al. 1992; Tassoni et al. 1992, 1999, 2000; Roldan and Bures 1994; Bielavska and Roldan 1996; Sacchetti et al. 1999, 2002; Bast et al. 2001). More importantly, several studies have shown that inactivating the amygdala with TTX disrupts conditioned fear. 
(Bucherelli et al. 1992; Tassoni et al. 1992; Sacchetti et al. 1999). Moreover, a recent study showed that reversible inactivation with TTX produces more deficits in conditioned fear than does reversible inactivation induced by the GABA-A agonist muscimol (Bast et al. 2001).

\section{MATERIALS AND METHODS}

\section{Experiment 1}

The purpose of the present experiment was to determine whether the shock-probe avoidance deficit observed with pretraining amygdala lesions reflects an inability to form an association between the shock and the probe. We hypothesized that if the amygdala is involved in shock-probe avoidance learning, then reversibly inactivating the amygdala with TTX immediately prior to exposure to an electrified probe should impair both acquisition and retention performance. Alternatively, if the amygdala is not critical for learning the association, but is involved in some other process that influences performance, then lesioned rats should not be impaired on the retention test when the effects of the lesions have dissipated.

\section{Methods}

All procedures were approved by the University of Alberta Biosciences Animal Policy and Welfare Committee and carried out in accordance with the guidelines of the Canadian Council on Animal Care (CCAC).

\section{Subjects}

Male Sprague Dawley rats (Ellerslie Laboratories; 250-300 g) were housed individually, kept on a 12:12 h light-dark cycle (lights on at 07:00 h), provided with food and water ad libitum, and allowed to acclimate to vivarium conditions for $1 \mathrm{wk}$ prior to the surgery.

\section{Surgical Procedures}

Rats were given the analgesic acetaminophen $(1.5 \mathrm{cc}, 100 \mathrm{mg} /$ $\mathrm{mL}$ ) orally. Approximately $1 \mathrm{~h}$ later they were given atropine sulfate $(0.2$ cc, $0.5 \mathrm{mg} / \mathrm{mL}$, i.p.; Ormond Veterinary Supply Ltd.) and anesthetized with sodium pentobarbital (50 mg/kg, i.p.; Abbott Laboratories Ltd.). Supplemental doses of sodium pentobarbital (25 mg/kg, i.p.; Abbott Laboratories Ltd.) were given as needed to maintain anesthesia. Once anesthetized, the rats were hydrated with $0.9 \%$ saline (3.0 cc, s.c.) and administered antibiotics (0.05 cc, i.m.; Penicillin G Procaine 300,000 i.u/mL; Rhône Mérieux Canada Inc.). The rats were then placed in a stereotaxic frame (Kopf Instruments), and a midline scalp incision was made to expose the top of the skull. Permanent stainless steel guide cannulae (23 gauge, $11 \mathrm{~mm}$ ) were implanted bilaterally with the tips aimed at the dorsal surface of the right and left amygdala (anterior-posterior $-2.3 \mathrm{~mm}$ from bregma, medial-lateral \pm 5.0 $\mathrm{mm}$ from midline, dorsal-ventral $-5.8 \mathrm{~mm}$ from dura, with the nose bar at $-3.2 \mathrm{~mm}$ from the interaural line; Paxinos and Watson 1997). The guide cannulae were affixed to the skull using four screws and dental acrylic. A stylet was inserted into the guide cannulae to keep them free from debris. The rats were allowed 1 wk to recover before behavioral testing commenced.

\section{Drugs and Infusion Procedure}

Five minutes before the shock-probe acquisition session, phosphate-buffered saline (VEHICLE; pH 7.4) or TTX (1 ng) was infused into the guide cannulae through injection needles (30 gauge; $12.5 \mathrm{~mm}$ ) attached to $10-\mu \mathrm{L}$ Hamilton syringes via polyethylene tubing (PE-50). The infusions $(0.5 \mu \mathrm{L})$ were delivered to both cannulae simultaneously over $60 \mathrm{sec}$ using a microinfusion pump (Harvard Apparatus). The injection needles were left in place for one additional minute following the infusions to maximize diffusion.

\section{Behavioral Procedure}

For the acquisition session, each rat was placed into the shockprobe apparatus for $15 \mathrm{~min}$. The apparatus consisted of a Plexiglas chamber $(40 \mathrm{~cm}$ long $\times 30 \mathrm{~cm}$ wide $\times 40 \mathrm{~cm}$ high $)$ with a wire-wrapped Plexiglas probe $(6.0 \times 0.5 \times 0.5 \mathrm{~cm})$ protruding from the center of one of the walls, $2 \mathrm{~cm}$ above the floor (Treit et al. 1993). For half the rats infused with VEHICLE or TTX, the probe was constantly electrified ( $2 \mathrm{~mA}$; SHOCK-EXPERIENCED), and for the other half, it was not (SHOCK-NAIVE). The total number of contact-induced shocks was measured in each rat exposed to the electrified probe and used as a measure of acquisition. Latency to the first contact-induced shock and the amount of time the rats spent immobile (resting, staying still) served as measures of general activity. In addition, the rats' behavioral reaction to each shock was scored according to a 4-point scale that ranges from a score of 1 for a flinch involving head or forepaw to a score of 4 for a whole body flinch and jump (all four feet in the air), followed by running to the opposite end of the chamber (Treit et al. 1993).

Four days later, retention performance was assessed in a second 15-min shock-probe session. The procedure was the same as for the acquisition session, with the exception that the rats were not infused with drug and the probe was electrified for all rats. The behavior of each rat was videotaped for both the acquisition and retention sessions. Furthermore, an observer unaware of the rat's prior drug and shock history scored the retention session.

\section{Histology}

Upon completion of behavioral testing, each rat was killed (chloral hydrate; $1 \mathrm{cc}, 800 \mathrm{mg} / \mathrm{mL}$, i.p.) and perfused intracardially with $0.9 \%$ saline followed by $10 \%$ formalin. The brains were stored in 10\% formalin for at least $48 \mathrm{~h}$ and then sectioned (60

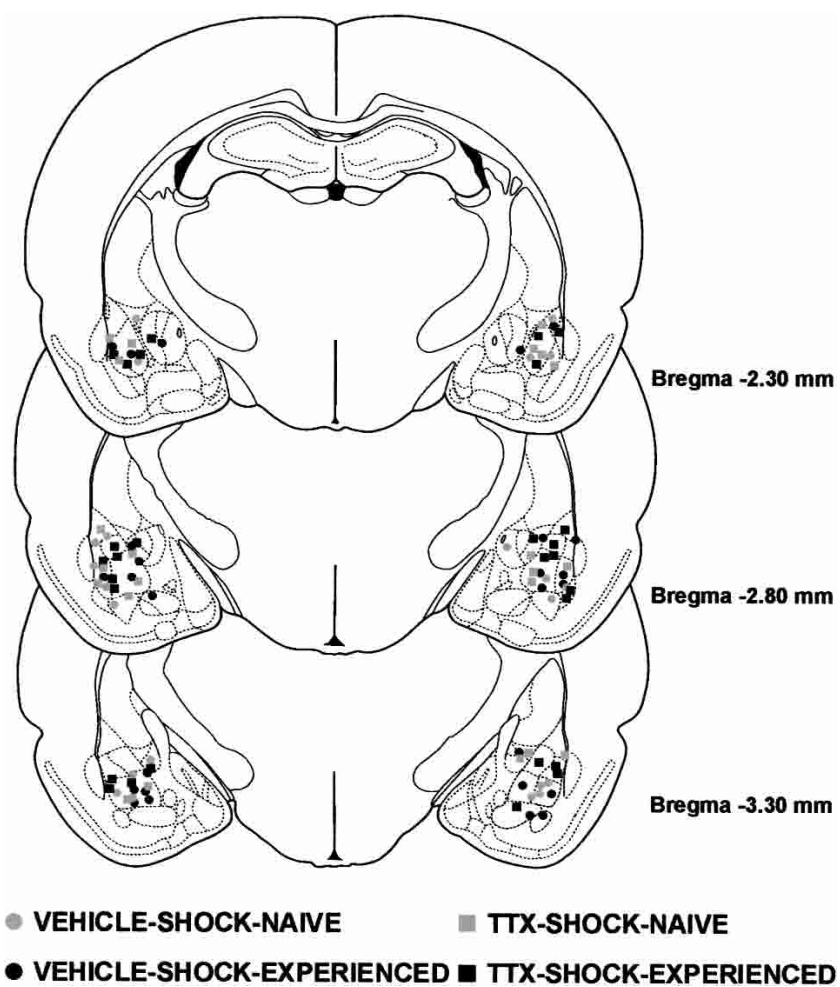

Figure 1 Illustrations of the infusion locations observed bilaterally through the rostral and caudal extent of the amygdala for the rats tested in Experiment 1. Atlas plates adapted from Paxinos and Watson (1997).

\section{Learning \& Memory}




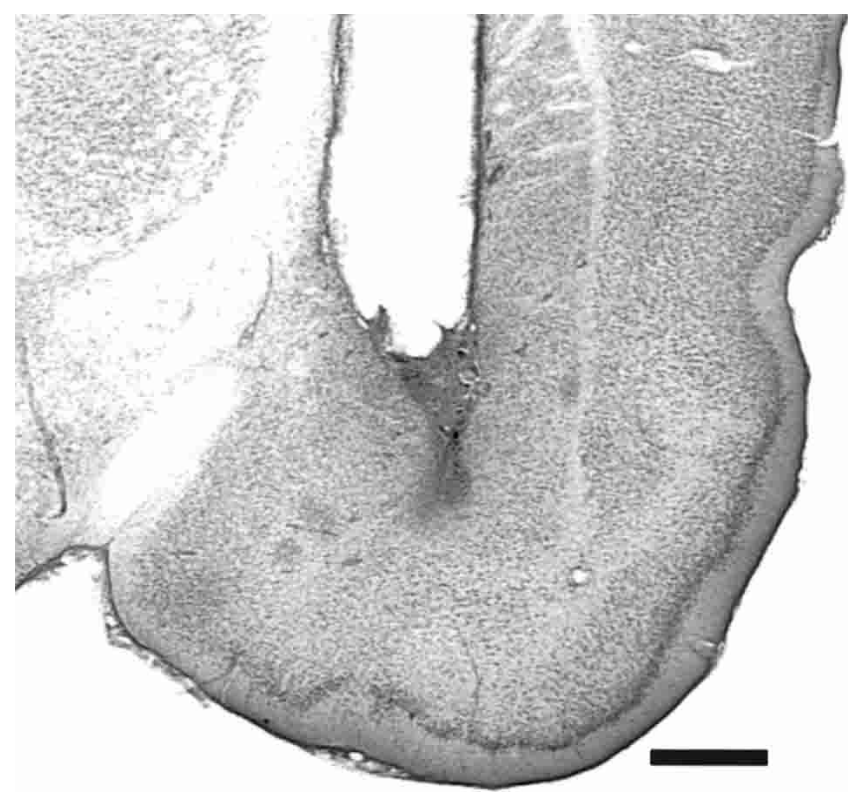

Figure 2 Photomicrograph of a coronal section of the temporal lobe showing a representative cannula placement $(-2.8 \mathrm{~mm}$ relative to bregma; Paxinos and Watson 1997). Scale bar, $1.0 \mathrm{~mm}$.

um), mounted on gelatin-coated slides, and stained with thionin. Each brain was analyzed for cannulae placement by an observer that was blind to the behavioral results and drug treatments. Cannula placements were considered acceptable if they were found within or immediately adjacent to the central or basolateral region of the amygdala. The behavioral results of rats with cannula placements that did not meet these criteria were excluded from the statistical analyses.

\section{Statistical Analysis}

Homogeneity of variance and normality of the distribution were violated for the latencies to first contact the probe during the acquisition session and the retention test. Consequently, these data were analyzed with the nonparametric Kruskal Wallis test and Mann-Whitney U-tests for post hoc comparisons where appropriate.

We also analyzed the other behavioral measures with the nonparametric Kruskal Wallis test and Mann-Whitney U-tests. Although the other behavioral measures typically did not violate any of the assumptions required for parametric analyses, the pattern of results did not change using either parametric or nonparametric tests. Hence, for ease of communication, nonparametric analyses were used in all instances.

\section{RESULTS}

\section{Histology}

The results of the histological analysis indicated that 49 rats met injection site placement criteria (Fig. 1). Figure 2 shows a photomicrograph of a representative cannula placement.

\section{Acquisition}

Inactivation of the amygdala impaired shock-probe avoidance in rats that were exposed to the electrified probe during the acquisition session (Fig. 3). Specifically, TTX-SHOCK-EXPERIENCED rats received significantly more contact-induced shocks than did VEHICLE-SHOCK-EXPERIENCED rats, $U=12.0, p<0.001$. This difference does not appear to be caused by differences in shock sensitivity, because there was no significant difference in shock reactivity between VEHICLE- and TTX-SHOCK-EXPERIENCED rats, $U=71.50, p=0.341$. Also, regardless of drug and shock manipulation, the general activity levels of the four groups were comparable because neither the latencies to first contact the probe, $X^{2}(3)=5.981, p=0.113$, nor the amount of time spent immobile differed between the groups, $X^{2}(3)=2.355, p=0.502$.

\section{Retention}

Figure 4 shows the results of the drug and shock manipulations on the shock-probe retention test. Inactivation of the amygdala during the acquisition session did not impair avoidance of the probe on the retention test, indicating that pretraining inactivation of the amygdala did not prevent the formation of an association between the shock and the probe. A significant difference was found between the groups, $X^{2}(3)=17.989, p<0.001$, but regardless of whether the amygdala was inactivated or not, the shock-probe avoidance of rats that were previously experienced with the electrified probe (SHOCK-EXPERIENCED) was significantly better than that of rats exposed to the nonelectrified probe (SHOCK-NAIVE), $p<0.001$. More specifically, VEHICLE-SHOCKEXPERIENCED rats had fewer contact-induced shocks $(p<0.005)$ than did VEHICLE-SHOCK-NAIVE rats. Similarly, compared with TTX-SHOCK-NAIVE rats, TTX-SHOCK-EXPERIENCED rats received significantly fewer contact-induced shocks $(p<0.005)$. In addition, the avoidance of TTX-SHOCK-EXPERIENCED rats did not differ from that of VEHICLE-SHOCK-EXPERIENCED rats $(p=0.243)$.

Amygdala-inactivated rats also successfully avoided the probe on the retention test when latencies to contact the probe were used as an index of memory. Again a significant difference between the groups was found, $X^{2}(3)=13.775, p<0.005$. Whether the amygdala was inactivated or not, the shock-probe avoidance of SHOCK-EXPERIENCED rats was significantly better

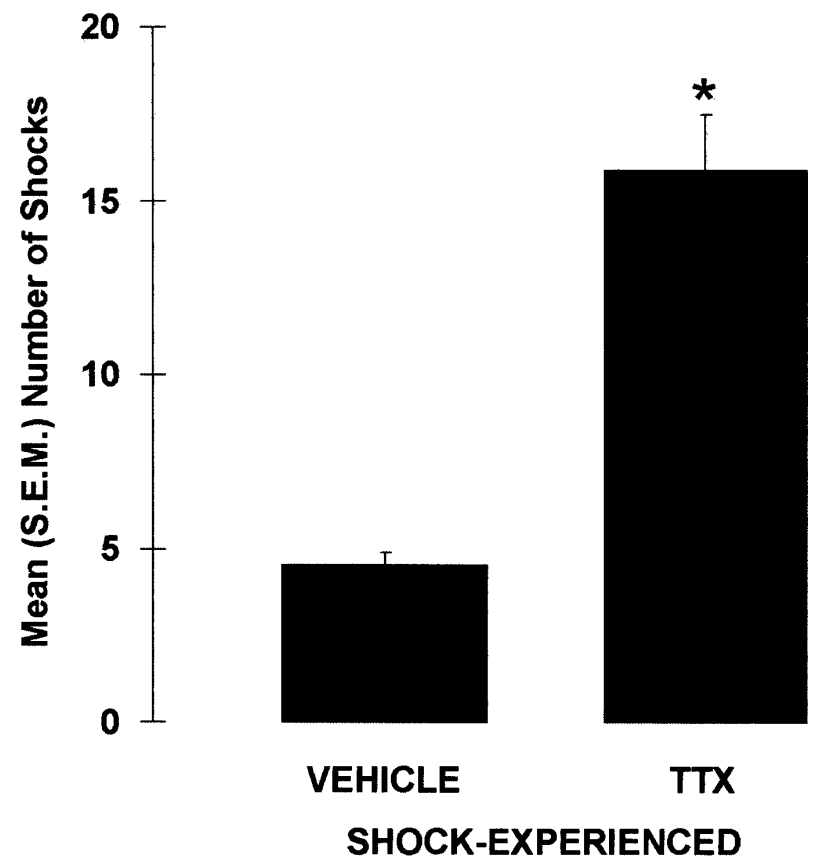

Figure 3 Mean $( \pm$ SEM) number of contact-induced shocks received during the acquisition session by rats immediately pretreated with either VEHICLE or TTX into the amygdala. $\left(^{*}\right) p<0.001$ versus VEHICLE; $n=13-$ 14 per group. 
A

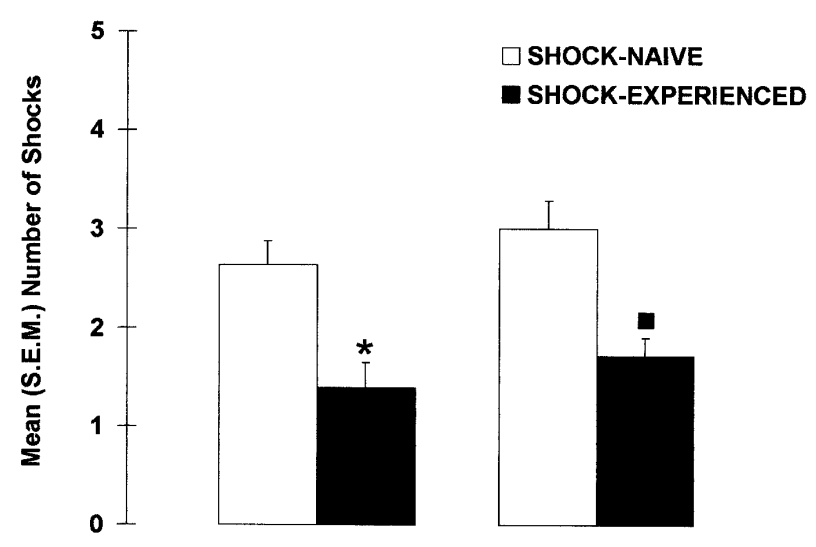

B
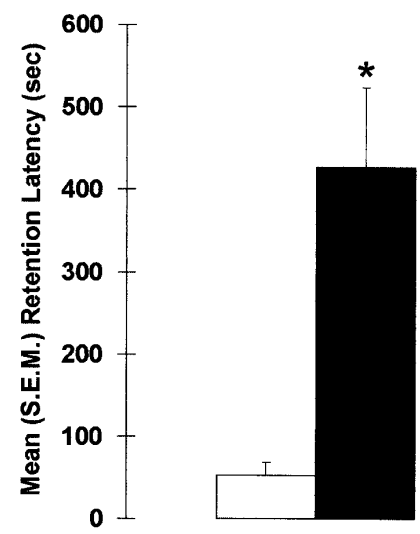

VEHICLE

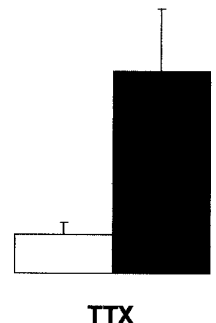

TTX

Figure 4 Mean $( \pm \operatorname{SEM})(A)$ number of contact-induced shocks and $(B)$ latency to the first contact-induced shock (retention latency) observed in rats that received pretraining intra-amygdala infusions of VEHICLE or TTX and were previously exposed to the electrified probe (SHOCK-EXPERIENCED) or not (SHOCK-NAIVE). ( $\left.{ }^{*}\right) p<0.01$ versus VEHICLE-SHOCKNAIVE; (ם) $p<0.01$ versus TTX-SHOCK-NAIVE; $n=11-14$ per group.

than that of SHOCK-NAIVE rats, $p<0.001$. More specifically, VEHICLE-SHOCK-EXPERIENCED rats had longer latencies than VEHICLE-SHOCK-NAIVE rats $(p<0.005)$, and TTX-SHOCK-EXPERIENCED rats tended to have significantly longer retention latencies than TTX-SHOCK-NAIVE rats $(p=0.063)$. Moreover, the retention latencies of VEHICLE- and TTX-SHOCK-EXPERIENCED rats did not significantly differ $(p=0.145)$, indicating that amygdala-lesioned rats avoided the electrified-probe as well as control rats.

The effects of manipulating shock experience and intraamygdala drug infusions on acquisition did not affect shock reactivity or general activity on the retention test. Neither shock reactivity, $X^{2}(3)=3.044, p=0.385$, nor the amount of time spent immobile, $X^{2}(3)=4.187, p=0.242$, differed between the groups.

\section{DISCUSSION}

Reversibly inactivating the amygdala immediately prior to a shock-probe acquisition session did not induce anterograde amnesia. On the retention test, the number of contact-induced shocks and the retention latencies did not differ between control and amygdala-lesioned rats. In addition, amygdala-lesioned rats received significantly less contact-induced shocks and tended to

have longer retention latencies than did lesioned naive rats. Although performance was not impaired in amygdala-lesioned rats on the retention test, their performance was impaired during the acquisition session. Compared with control rats, amygdala-lesioned rats received more contact-induced shocks during the shock-probe acquisition session.

Taken together, these findings indicate that the shock-probe avoidance deficits caused by pretraining amygdala lesions do not reflect an inability to form an association between the shock and the probe. If the lesions had prevented the formation of this association, then the lesions should have impaired both acquisition and retention performance. Consequently, the deficits observed during the acquisition session likely reflect the involvement of the amygdala in some process other than learning that influences performance. For example, the lesions may reduce anxiety levels, which could account for the increase in the number of contact-induced shocks observed in amygdala-inactivated rats during the acquisition session. This interpretation would be consistent with other findings that have implicated the amygdala in unconditioned fear and anxiety (Blanchard and Blanchard 1972; Dunn and Everitt 1988; Kemble et al. 1990; Kesner et al. 1992; Pesold and Treit 1994; Burns et al. 1996; Vazdarjanova et al. 2001).

\section{MATERIALS AND METHODS}

\section{Experiment 2}

Although the results of Experiment 1 indicate that the amygdala is not critical for shock-probe avoidance learning, it remains possible that the spared memory observed in amygdala-lesioned rats was caused by overtraining. During the acquisition session of Experiment 1, amygdala-lesioned rats received four times as many shocks as did control rats. This increase in the number of contact-induced shocks may have engaged other neural structures, and these structures may be responsible for the spared retention that was observed in the amygdala-lesioned rats. Several studies have found that overtraining rats in conditioned fear paradigms reduces the memory deficits caused by amygdala lesions (Parent et al. 1992, 1994, 1995b; Kim and Davis 1993; Lee et al. 1996; Maren 1998, 1999).

To determine whether overtraining during the acquisition session accounted for the spared memory on the retention test, we examined the effects of limiting the number of contact-induced shocks rats received during acquisition. Specifically, in Experiment 2 we repeated the procedure used in Experiment 1, with the exception that both Vehicle and TTX rats were limited to four shocks during the acquisition session. This is the number of shocks that vehicle-infused rats received during the acquisition session in Experiment 1.

We hypothesized that if the amygdala is involved in shockprobe avoidance learning, then pretraining intra-amygdala infusions of TTX should impair shock-probe performance on the retention test. Alternatively, if the amygdala is not critical for shock-probe avoidance learning, then the retention performance of rats infused with either Vehicle or TTX prior to training should not differ on the retention test. Such a finding would indicate that overtraining did not account for the spared shock-probe learning observed in amygdala-lesioned rats in Experiment 1.

\section{Methods}

\section{Procedures}

The same procedures as Experiment 1 were used, with the exception that for the acquisition session, each rat was placed in the shock-probe apparatus for a maximum of four contacts with the probe (whether electrified or not) or a maximum of $15 \mathrm{~min}$, whichever came first. 


\section{RESULTS}

\section{Histology}

The results of the histological analysis indicated that 46 rats met infusion site criteria (Fig. 5). The histological criteria were the same as in Experiment 1.

\section{Acquisition}

All of the rats exposed to the electrified probe received a total of four shocks, with the exception of one VEHICLE-SHOCK-EXPERIENCED rat that received two shocks. However, the mean number of contact-induced shocks did not vary significantly between the VEHICLE-SHOCK-EXPERIENCED $(M=3.714)$ and the TTXSHOCK-EXPERIENCED rats $(M=4.0), U=42.0, p=0.157$. Also, shock reactivity in TTX-SHOCK-EXPERIENCED rats $(M=1.809)$ was significantly lower than that of VEHICLE-SHOCK-EXPERIENCED rats $(M=2.143), U=10.500, p<0.005$.

None of the groups differed in the initial latency to come in contact with the probe $X^{2}(3)=5.069, p=0.167$. However, the overall amount of time spent in the apparatus differed between the groups, $X^{2}(3)=10.684, p<0.05$. VEHICLE-SHOCK-EXPERIENCED rats remained in the apparatus for a greater amount of time than any other group ( $p s<0.05)$, whereas the other groups did not differ from each other (all $p s>0.05$ ). The amount of time spent immobile also differed between the groups $X^{2}(3)=23.816$, $p<0.001$. This was because of the finding that the VEHICLESHOCK-EXPERIENCED rats spent more time immobile than any other group ( $p s<0.01$ ), but the other groups did not significantly differ from each other (all $p s>0.05$ ).

\section{Retention}

Figure 6 shows the results of the drug and shock manipulations on the shock-probe retention test. Inactivation of the amygdala

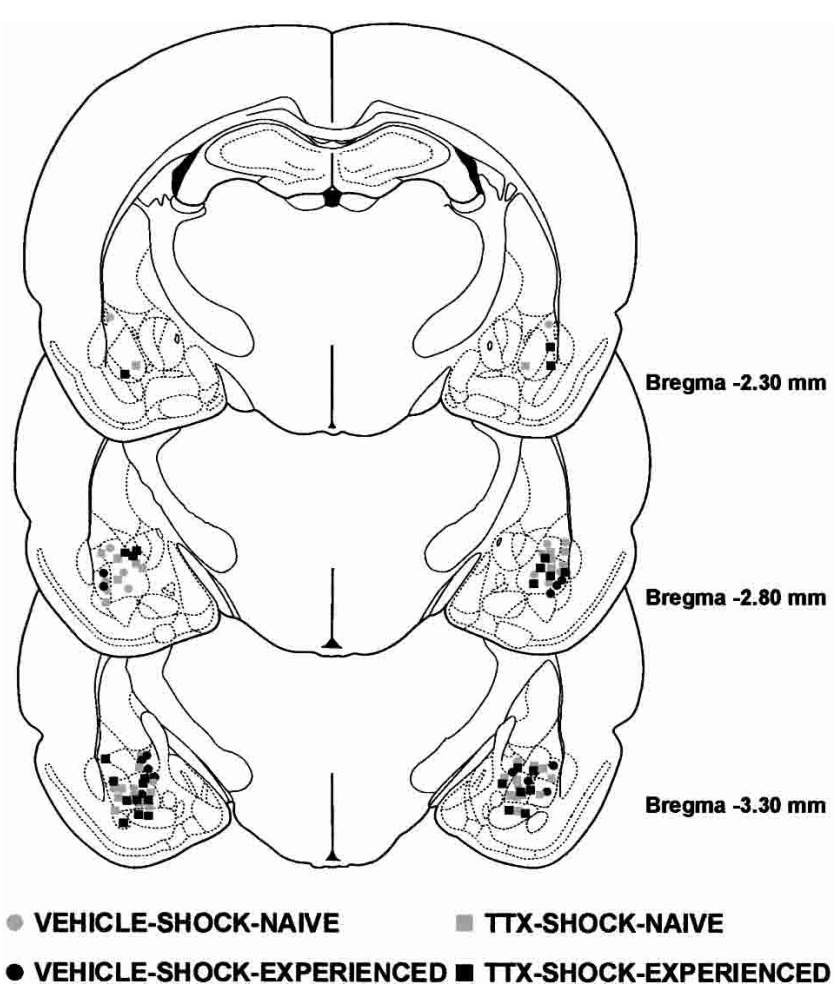

Figure 5 Illustrations of the infusion locations observed bilaterally through the rostral and caudal extent of the amygdala for the rats tested in Experiment 2. Atlas plates adapted from Paxinos and Watson (1997). during the shock-probe acquisition session did not impair avoidance of the probe on the retention test, indicating that pretraining inactivation of the amygdala did not prevent the formation of an association between the shock and the probe. A significant difference was found between the groups for the number of contact-induced shocks, $X^{2}(3)=14.800, p<0.005$, but this difference was not caused by the effect of the amygdala inactivation. Even though the rats were limited to a maximum of four contacts with the probe during the acquisition session, SHOCK-EXPERIENCED rats received fewer contact-induced shocks than did SHOCK-NAIVE rats on the retention test $(p<0.001)$. VEHICLE SHOCK-EXPERIENCED rats received fewer contact-induced shocks than VEHICLE-SHOCK-NAIVE rats $(p<0.01)$. Likewise, TTX-SHOCK-EXPERIENCED rats received significantly fewer shocks than did TTX-SHOCK-NAIVE rats $(p<0.05)$. In addition, the shock avoidance of TTX-SHOCK-EXPERIENCED rats was as good as that of VEHICLE SHOCK-EXPERIENCED rats because the number of contact-induced shocks did not differ between these two groups $(p=0.312)$.

A similar avoidance pattern was found when considering the retention latencies. The latencies to contact the probe on the retention test differed between the groups, $X^{2}(3)=11.981$, $p<0.01$. SHOCK-EXPERIENCED rats took longer to contact the probe than did SHOCK-NAIVE rats $(p<0.005)$. More specifically, VEHICLE SHOCK-EXPERIENCED rats had longer latencies than VEHICLE-SHOCK-NAIVE rats $(p<0.05)$, and TTX-SHOCK-EXPERIENCED rats had significantly longer retention latencies than TTX-SHOCK-NAIVE rats $(p<0.05)$. Moreover, the retention latencies of VEHICLE- and TTX-SHOCK-EXPERIENCED rats did not significantly differ $(p=0.191)$, indicating that amygdala-lesioned rats avoided the electrified probe as well as control rats.

The drug and shock manipulation did not affect shock sensitivity or activity levels on the retention test. There were no significant differences between the groups for shock reactivity, $X^{2}(3)=0.435, p=0.933$, or the amount of time spent immobile, $X^{2}(3)=1.009, p=0.799$, on the retention test.

\section{DISCUSSION}

The findings of the present experiments demonstrate that reversibly inactivating the amygdala immediately before a shock-probe acquisition session does not cause anterograde amnesia for the association between the probe and the shock. Experiment $1 \mathrm{dem}-$ onstrated that reversibly lesioning the amygdala before rats were trained in the shock-probe paradigm did not impair avoidance of the probe on a retention test given $4 \mathrm{~d}$ later. Specifically, the retention performance of amygdala-lesioned rats was comparable to that of control rats and better than that of lesioned shocknaive rats. The results of Experiment 2 demonstrated that the spared shock-probe retention was not caused by overtraining. When lesioned rats were limited to four shocks during acquisition (i.e., control levels), they also did not show any deficits on the retention test.

Although inactivation of the amygdala did not impair retention performance, it did have a profound effect on behavior during the acquisition session. The results of Experiment 1 showed that inactivation of the amygdala immediately before a first shock-probe session dramatically increased the number of shocks rats received during that session. This replicates the findings of several studies showing that pretraining lesions of the amygdala impair shock-probe avoidance (Kopchia et al. 1992; Treit et al. 1993; Treit and Menard 1997; Lehmann et al. 2000).

Combined, these findings indicate that pretraining amygdala lesions do not prevent the formation of an association between the shock and the probe. If the amygdala were involved in the formation of this association, then the amygdala lesions 
A

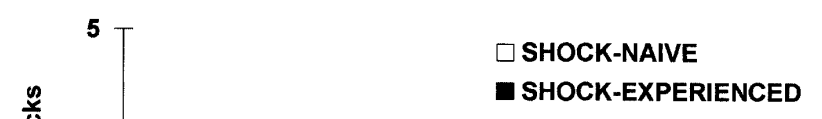

B
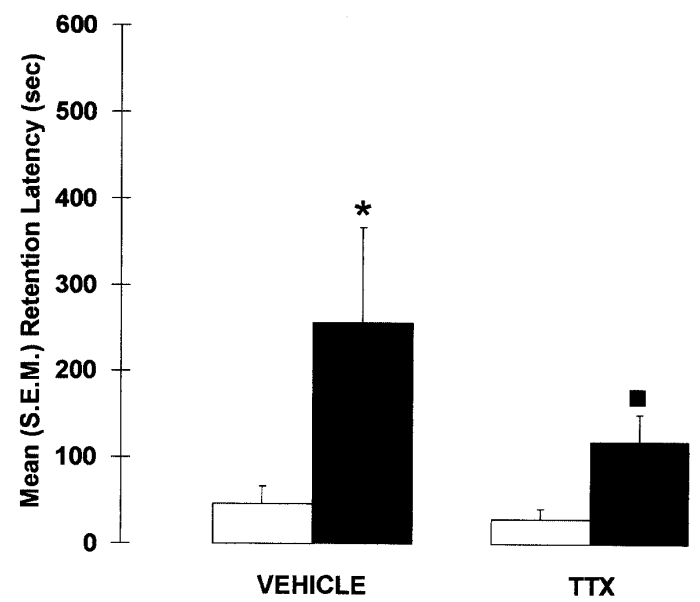

TTX

Figure 6 Mean $( \pm \operatorname{SEM})(A)$ number of contact-induced shocks and $(B)$, latency to the first contact-induced shock (retention latency) observed in rats that received pretraining intra-amygdala infusions of VEHICLE or TTX and were previously limited to four contacts with the electrified probe (SHOCK-EXPERIENCED) or the nonelectrified probe (SHOCK-NAIVE) during the acquisition session. $\left(^{*}\right) p<0.05$ versus VEHICLE-SHOCK-NAIVE; (घ) $p<0.05$ versus TTX-SHOCK-NAIVE; $n=7-15$ per group.

should have impaired both acquisition and retention performance. These findings are similar to those of other studies investigating the role of the amygdala in fear conditioning. For instance, Vazdarjanova and McGaugh (1998) found that pretraining lesions of the basolateral complex in rats did not block avoidance learning in a contextual fear-conditioning task. Sutherland and McDonald (1990) as well as Vanderwolf et al. (1988) have also shown that the amygdala is not necessary for the acquisition of contextual fear conditioning when the mean amount of defecation is used as an index of learning and memory. In addition, two studies that examined the effects of reversible amygdala lesions also revealed that the amygdala might not be necessary for the acquisition of conditioned fear as indexed by freezing (Helmstetter 1992; Helmstetter and Bellgowan 1994). However, the authors of these latter two studies raise the possibility that acquisition may not have been impaired in these studies owing to the pharmacological characteristics of the lidocaine and muscimol that were used to inactivate the amygdala.

The finding that amygdala-lesioned rats demonstrated memory on the retention test in the present study automatically implies that associative encoding was not blocked by inactivation of the amygdala during the acquisition session. The finding by others that amygdala-lesioned rats bury the electrified probe during shock-probe training also indicates that they are able to learn in the shock-probe paradigm (Roozendaal et al. 1991; Kopchia et al. 1992; Treit et al. 1993; Treit and Menard 1997). Similar findings have been observed in contextual fear conditioning (Antoniadis and McDonald 2001; Holahan and White 2002). For instance, Antoniadis and McDonald (2001) found that pretraining amygdala lesions did not block the freezing response during contextual fear conditioning, although performance was impaired on the retention test.

This successful associative encoding in the amygdala-lesioned rats indicates that the impaired avoidance observed during the acquisition session likely reflects the involvement of the amygdala in a nonmnemonic process that influences performance. For example, extensive evidence indicates that the amygdala is involved in unconditioned fear and anxiety (Blanchard and Blanchard 1972; Dunn and Everitt 1988; Kemble et al. 1990; Kesner et al. 1992; Pesold and Treit 1994; Burns et al. 1996; Vazdarjanova et al. 2001). The possibility that the avoidance deficit observed during the acquisition session reflects the amygdala's involvement in the unconditioned fear necessary for probe avoidance is supported further by the finding that amygdala lesions also do not affect the expression of conditioned shock avoidance. Specifically, Lehmann et al. (2000) found that rats given permanent neurotoxic amygdala lesions following shockprobe training were able to avoid the probe when they were re-exposed to it. Avoidance deficits were only observed in lesioned rats whose first exposure to the electrified probe occurred after the induction of the lesion. This result is analogous to the findings of File et al. (1993). These researchers found that diazepam does not reduce anxiety in the elevated plus-maze in rats that have had prior experience in the apparatus, whereas diazepam has robust anxiolytic effects when rats are naive to the maze. They interpreted their findings as indicating that the plusmaze can elicit two types of fear: an unconditioned or innate fear and a learned fear. Similarly, in the shock-probe paradigm, amygdala lesions may have impaired an innate fear to the electrified probe, as seen by the increased number of shocks during the first exposure, but may not have affected learned fear as indicated by avoidance of the probe upon re-exposure to it.

Although pretraining amygdala lesions impair initial shockprobe avoidance, several studies have reported that amygdala lesions do not impair burying of the electrified probe. Amygdalalesioned rats bury the electrified probe as much and in some instances more than control rats (Roozendaal et al. 1991; Kopchia et al. 1992; Treit et al. 1993; Treit and Menard 1997). Also, lesioned rats display normal increases in heart rate upon experience with the electrified probe (Roozendaal et al. 1991). These findings indicate that the lesioned rats are capable of forming an association between the shock and the probe, but that the expression of this association is response-specific. Furthermore, these findings support the possibility that fear may not be a unified construct, but, rather, that there are different types of fear responses, which may be controlled by different brain structures (Pesold and Treit 1994).

It is possible that the infusions of TTX into the amygdala during the acquisition sessions did not induce anterograde amnesia because the TTX did not completely block amygdala function. However, there are numerous lines of evidence that indicate that this possibility is unlikely. TTX is a sodium channel blocker that has been shown to be very efficient for reversibly inactivating neural structures (see Ambrogi Lorenzini et al. 1997). Several studies have shown that similar infusions of TTX into the amygdala produce robust behavioral effects (Bucherelli et al. 1992; 
Tassoni et al. 1992; Roldan and Bures 1994; Bielavska and Roldan 1996). Others have shown that a concentration of TTX that is lower than that used in the present study is sufficient to induce amnesia (Roldan and Bures 1994). In the present study, the TTX was delivered in a volume of $0.5 \mu \mathrm{L}$, which has been shown previously to spread through most of the amygdala (Izquierdo et al. 1997). Furthermore, studies that have used this volume to target the amygdala with other drugs have shown mnemonic enhancement or impairments (Packard et al. 1994; Izquierdo et al. 1997; Packard and Teather 1998). As Figure 1 shows, our cannulae were directed primarily toward the basolateral complex of the amygdala, which is the region of the amygdala that is considered to be critical for fear conditioning (see Fanselow and LeDoux 1999). Given the cannula placement, the injection volume, and the concentration of TTX, it is highly probable that the TTX produced complete inactivation of the basolateral region of the amygdala. Finally, the present results showed that the injections of TTX into the amygdala had a marked effect on the number of shocks received during the acquisition session of Experiment 1 , which supports our contention that the infusions were effective.

It is also important to note that the absence of amnesia following amygdala inactivation was not caused by a decrease in activity levels, because there were no differences in time spent immobile in any of the retention conditions. The spared memory was also not caused by a lesion-induced increase in shock sensitivity. In neither experiment did amygdala-lesioned rats react more than control rats to the shocks. In Experiment 2, however, amygdala-inactivated rats did react less to the shock during the acquisition session. This latter finding is unusual given that, to our knowledge, amygdala lesions have never significantly decreased shock-probe reactivity (Treit et al 1993; Treit and Menard 1997; Lehmann et al. 2000). This inconsistent finding may be due to chance or may indicate that amygdala lesions decrease pain thresholds for the first contact-induced shocks. Regardless, this reduction in shock reactivity in amygdala-inactivated rats would have decreased, rather than increased the likelihood of learning the association between the shock and the probe.

Similarly, the spared memory in Experiment 2 was not due to differences in the temporal distribution of the shocks between control and lesioned rats. In Experiment 2, control rats exposed to the electrified probe remained in the apparatus longer than did amygdala-inactivated rats exposed to the electrified probe. Given that both groups were limited to four shocks, this indicates that amygdala-lesioned rats massed their shocks compared with control rats. However, this massed training could not account for the spared memory, because extensive evidence indicates that massed learning is not as efficient as distributed learning (Fanselow and Tighe 1988; Williams et al. 1991; Fanselow et al. 1993; Yin et al. 1994).

Although inactivation of the amygdala during the acquisition session did not affect the number of shocks the TTX rats received on the retention test, it did tend to reduce their retention latencies. Specifically, in both Experiments 1 and 2, the latencies to contact the probe on the retention test tended to be shorter for the amygdala-lesioned rats than for the control rats. It is possible that this tendency for reduced latencies is indicative of a mild amnesic effect of TTX that reflects the involvement of the amygdala in learning the association between the probe and the shock. Alternatively, it may have resulted from the loss of amygdala modulatory signals during the consolidation phase. Given the time course of TTX effects (Zhuravin and Bures 1991; Ambrogi Lorenzini et al. 1997), the functional block produced by the pretraining infusions of TTX likely persisted during the postlearning period and extensive evidence indicates that the amygdala regulates memory storage processes during this consolida- tion phase (McGaugh 2002). For example, several studies have shown that reversible inactivation of the amygdala immediately after training impairs performance in conditioned fear tasks (Jerusalinsky et al. 1994; Parent and McGaugh 1994; Izquierdo et al. 1997; Sacchetti et al. 1999; Vazdarjanova and McGaugh 1999). Similarly, immediate posttraining intraamygdala injections of a variety of agents acting at different neurotransmitter systems modulate memory in shock-motivated tasks (Brioni et al. 1989; Izquierdo et al. 1992; Introini-Collison et al. 1996; Roozendaal and McGaugh 1997; Vazdarjanova and McGaugh 1999; Wilensky et al. 2000).

There are many processes that likely contribute to successful shock-probe avoidance. Although avoidance of the probe is an instrumental response, successful probe avoidance likely involves both Pavlovian and instrumental conditioning. To effectively avoid the probe, the rat must first form a Pavlovian association between the probe and the sensation of the shock. This would then be followed by the formation of an instrumental association between the probe and the avoidance response. It is possible that effective avoidance of the probe may occur through the formation of associations that ultimately do not involve fear. On the other hand, it has been suggested that knowing that a stimulus is aversive in the absence of experiencing emotion is not sufficient for successful avoidance (Bechara et al. 1999).

In summary, the present findings demonstrate that pretraining reversible inactivation of the amygdala does not produce retention deficits in a shock-probe avoidance task. Combined with the finding that the lesions do impair performance during shockprobe training, these findings indicate that the amygdala is not necessary for the acquisition of the association between the shock and the cue, but is involved in some other process that influences performance.

\section{ACKNOWLEDGMENTS}

We are grateful for the technical assistance provided by Vishnu Singh as well as comments from Melissa Glenn on an earlier version of this manuscript. This work was supported by NSERC OGP0038726 (to D.T.) and NSERC OGP019453 NINDS, NIDDK, JDK 1RO1 NS 41173-01 (to M.B.P.).

The publication costs of this article were defrayed in part by payment of page charges. This article must therefore be hereby marked "advertisement" in accordance with 18 USC section 1734 solely to indicate this fact.

\section{REFERENCES}

Ambrogi Lorenzini, C.G., Baldi, E., Bucherelli, C., Sacchetti, B., and Tassoni, G. 1997. Analysis of mnemonic processing by means of totally reversible neural inactivations. Brain Res. Brain Res. Protoc. 1: 391-398.

Antoniadis, E.A. and McDonald, R.J. 2001. Amygdala, hippocampus, and unconditioned fear. Exp. Brain Res. 138: 200-209.

Bast, T., Zhang, W.N., and Feldon, J. 2001. The ventral hippocampus and fear conditioning in rats. Different anterograde amnesias of fear after tetrodotoxin inactivation and infusion of the GABA(A) agonist muscimol. Exp. Brain Res. 139: 39-52.

Bechara, A., Damasio, H., Damasio, A.R., and Lee, G.P. 1999. Different contributions of the human amygdala and ventromedial prefrontal cortex to decision-making. J. Neurosci. 19: 5473-5481.

Bermudez-Rattoni, F., Introini-Collison, I., Coleman-Mesches, K., and McGaugh, J.L. 1997. Insular cortex and amygdala lesions induced after aversive training impair retention: Effects of degree of training. Neurobiol. Learn. Mem. 67: 57-63.

Bielavska, E. and Roldan, G. 1996. Ipsilateral connections between the gustatory cortex, amygdala and parabrachial nucleus are necessary for acquisition and retrieval of conditioned taste aversion in rats. Behav. Brain Res. 81: 25-31.

Blanchard, D.C. and Blanchard, R.J. 1972. Innate and conditioned reactions to threats in rats with amygdaloid lesions. J. Comp. Physiol. Psychol. 81: 281-290.

Brioni, J.D., Nagahara, A.H., and McGaugh, J.L. 1989. Involvement of 
the amygdala gabaergic system in the modulation of memory storage. Brain Res. 487: 105-112.

Bucherelli, C., Tassoni, G., and Bures, J. 1992. Time-dependent disruption of passive avoidance by post-training intra-amygdala injection of tetrodotoxin in rats. Neurosci. Lett. 140: 231-234.

Burns, L.H., Annett, L., Kelley, A.E., Everitt, B.J., and Robbins, T.W 1996. Effects of lesions to amygdala, ventral subiculum, medial prefrontal cortex, and nucleus accumbens on the reaction to novelty: Implication for limbic-stratial interactions. Behav. Neurosci. 110: $60-73$.

Cahill, L., Vazdarjanova, A., and Setlow, B. 2000. The basolateral amygdala complex is involved in, but not necessary for, rapid acquisition of Pavlovian fear conditioning. Eur. J. Neurosci. 12: $3044-3050$

Dunn, L.T. and Everitt, B.J. 1988. Double dissociations of the effects of amygdala and insular cortex lesions on conditioned taste aversion, passive avoidance, and neophobia in the rat using the excitotoxin ibotenic acid. Behav. Neurosci. 102: 3-23.

Fanselow, M.S. and LeDoux, J.E. 1999. Why we think plasticity underlying Pavlovian fear conditioning occurs in the basolateral amygdala. Neuron 23: 229-232.

Fanselow, M.S. and Tighe, T.J. 1988. Contextual conditioning with massed versus distributed unconditional stimuli in the absence of explicit conditional stimuli. J. Exp. Psychol. Anim. Behav. Process. 14: $187-199$.

Fanselow, M.S., DeCola, J.P., and Young, S.L. 1993. Mechanisms responsible for reduced contextual conditioning with massed unsignaled unconditional stimuli. J. Exp. Psychol. Anim. Behav. Process. 19: 121-137.

File, S.E., Zangrossi, H., Viana, M., and Graeff, F.G. 1993. Trial 2 in the elevated plus-maze: A different form of fear? Psychopharmacology 111: 491-494.

Helmstetter, F.J. 1992. Contribution of the amygdala to learning and performance of conditioned fear. Physiol. Behav. 51: 1271-1276.

Helmstetter, F.J. and Bellgowan, P.S. 1994. Effects of muscimol applied to the basolateral amygdala on acquisition and expression of contextual fear conditioning in rats. Behav. Neurosci. 108: 10051009.

Holahan, M.R. and White, N.M. 2002. Conditioned memory modulation, freezing, and avoidance as measures of amygdala-mediated fear. Neurobiol. Learn. Mem. 77: 250-275.

Introini-Collison, I.B., Dalmaz, C., and McGaugh, J.L. 1996. Amygdala $\beta$-noradrenergic influences on memory storage involve cholinergic activation. Neurobiol. Learn. Mem. 1: 57-64.

Izquierdo, I., da Cunha, C., Rosat, R., Jerusalinsky, D., Ferreira, M.B., and Medina, J.H. 1992. Neurotransmitter receptors involved in post-training memory processing by the amygdala, medial septum, and hippocampus of the rat. Behav. Neural Biol. 1: 16-26.

Izquierdo, I., Quillfeldt, J.A., Zanatta, M.S., Quevedo, J., Schaeffer, E., Schmitz, P.K., and Medina, J.H. 1997. Sequential role of hippocampus and amygdala, entorhinal cortex and parietal cortex in formation and retrieval of memory for inhibitory avoidance in rats. Eur. I. Neurosci. 9: 786-793.

Jerusalinsky, D., Ferreira, M.B.C., Walz, R., da Silva, R.C., Bianchin, M., Ruschel, A.C., Zanatta, M.S., Medina, J.H., and Izquierdo, I. 1994. Amnesia by post-training infusions of glutamate receptor antagonists into the amygdala, hippocampus, and entorhinal cortex. Behav. Neural Biol. 58: 76-80.

Kemble, E.D., Blanchard, D.C., and Blanchard, R.J. 1990. Effects of regional amygdaloid lesions on flight and defensive behaviors of wild black rats (Rattus rattus). Physiol. Behav. 48: 1-5.

Kesner, R.P., Berman, R.F., and Tardif, R. 1992. Place and taste learning: Role of basal forebrain, parietal cortex, and amygdala. Brain Res. Bull. 29: 345-353.

Killcross, S., Robbins, T.W., and Everitt, B.J. 1997. Different types of fear-conditioned behavior mediated by separate nuclei within amygdala. Nature 388: 377-380.

Kim, M. and Davis, M. 1993. Electrolytic lesions of the amygdala block acquisition and expression of fear-potentiated startle even with extensive training but do not prevent reacquisition. Behav. Neurosci. 107: 580-595.

Kopchia, K.L., Altman, H.J., and Commissaris, R.L. 1992. Effects of lesions of the central nucleus of the amygdala on anxiety-like behaviors in the rat. Pharmacol. Biochem. Behav. 43: 453-461.

LaBar, K.S. and LeDoux, J.E. 1996. Partial disruption of fear conditioning in rats with unilateral amygdala damage: Correspondence with unilateral temporal lobectomy in humans. Behav. Neurosci. 110: 991-997.

Lee, Y., Walker, D., and Davis, M. 1996. Lack of a temporal gradient of retrograde amnesia following NMDA-induced lesions of the basolateral amygdala assessed with the fear-potentiated startle paradigm. Behav. Neurosci. 110: 836-839.
Lehmann, H., Treit, D., and Parent, M.B. 2000. Amygdala lesions do not impair shock-probe avoidance retention performance. Behav. Neurosci. 114: 107-116.

Liang, K.C., McGaugh, J.L., Martinez, J.L., Jensen, R.A., Vasquez, B.J., and Messing, R.B. 1982. Post-training amygdaloid lesions impair retention of an inhibitory avoidance response. Behav. Brain Res. 4: $237-249$.

Maren, S. 1998. Overtraining does not mitigate contextual fear conditioning deficits produced by neurotoxic lesions of the basolateral amygdala. J. Neurosci. 18: 3088-3097.

. 1999. Neurotoxic basolateral amygdala lesions impair learning and memory but not the performance of conditional fear in rats. $J$. Neurosci. 19: 8696-8703.

Maren, S., Aharonov, G., and Fanselow, M.S. 1996. Retrograde abolition of conditional fear after excitotoxic lesions in the basolateral amygdala of rats: Absence of a temporal gradient. Behav. Neurosci. 110: $718-726$

McGaugh, J.L. 2002. Memory consolidation and the amygdala: A systems perspective. Trends Neurosci. 25: 456-461.

Muller, J., Corodimas, K.P., Fridel, Z., and LeDoux, J.E. 1997. Functional inactivation of the lateral and basal nuclei of the amygdala by muscimol infusion prevents fear conditioning to an explicit conditioned stimulus and contextual stimulus. Behav. Neurosci. 111: 683-691.

Packard, M.G. and Teather, L.A. 1998. Amygdala modulation of multiple memory systems: Hippocampus and caudate-putamen. Neurobiol. Learn. Mem. 69: 163-203.

Packard, M.G., Cahill, L., and McGaugh, J.L. 1994. Amygdala modulation of hippocampal-dependent and caudate nucleus-dependent memory processes. Eur. J. Neurosci. 91: $8477-8481$.

Parent, M.B. and McGaugh, J.L. 1994. Posttraining infusion of lidocaine into the amygdala basolateral complex impairs retention of inhibitory avoidance training. Brain Res. 661: 97-103.

Parent, M.B., Tomaz, C., and McGaugh, J.L. 1992. Increased training in an aversively motivated task attenuates the memory-impairing effects of posttraining $N$-methyl-D-aspartate-induced amygdala lesions. Behav. Neurosci. 106: 791-799.

Parent, M.B., West, M., and McGaugh, J.L. 1994. Memory of rats with amygdala lesions induced 30 days after footshock-motivated escape training reflects degree of original training. Behav. Neurosci. 108: $1080-1087$.

Parent, M.B., Avila, E., and McGaugh, J.L. 1995a. Footshock facilitates the expression of aversively motivated memory in rats given post-training amygdala basolateral complex lesions. Brain Res. 676: $235-244$

Parent, M.B., Quirarte, G.L., Cahill, L., and McGaugh, J.L. 1995b. Spared retention of inhibitory avoidance learning after posttraining amygdala lesions. Behav. Neurosci. 109: 803-807.

Paxinos, G. and Watson, C. 1997. The rat brain in stereotaxic coordinates, Compact 3rd ed. Academic Press, San Diego, CA.

Pesold, C. and Treit, D. 1994. The septum and amygdala differentially mediate the anxiolytic effects of benzodiazepines. Brain Res. 638: 295-301.

Phillips, R.G. and LeDoux, J.E. 1992. Differential contribution of amygdala and hippocampus to cued and contextual fear conditioning. Behav. Neurosci. 106: 274-285.

Poremba, A. and Gabriel, M. 1999. Amygdala neurons mediate acquisition but not maintenance of instrumental avoidance behavior in rabbits. J. Neurosci. 19: 9635-9641.

Roldan, J. and Bures, G. 1994. Tetrodotoxin blockade of amygdala overlapping with poisoning impairs acquisition of conditioned taste aversion in rats. Behav. Brain Res. 15: 213-219.

Roozendaal, B. and McGaugh, J.L. 1997. Glucocorticoid receptor agonist and antagonist administration into the basolateral but not central amygdala modulates memory storage. Neurobiol. Learn. Mem. 67: $176-179$.

Roozendaal, B., Koolhaas, J.M., and Bohus, B. 1991. Central amygdala lesions affect behavioral and autonomic balance during stress in rats. Physiol. Behav. 50: 777-781.

Sacchetti, B., Lorenzini, C.A., Baldi, E., Tassoni, G., and Bucherelli, C. 1999. Auditory thalamus, dorsal hippocampus, basolateral amygdala, and perirhinal cortex role in the consolidation of conditioned freezing to context and to acoustic conditioned stimulus in the rat. J. Neurosci. 19: 9570-9578.

Sacchetti, B., Baldi, E., Lorenzini, C.A., and Bucherelli, C. 2002. Differential contribution of some cortical sites to the formation of memory traces supporting fear conditioning. Exp. Brain Res. 146: $223-232$.

Sananes, C.B. and Davis, M. 1992. N-Methyl-D-aspartate lesions of the lateral and basolateral nuclei of the amygdala block fear-potentiated startle and shock sensitization of startle. Behav. Neurosci. 106: 72-80.

\section{Learning \& Memory


Sutherland, R.J. and McDonald, R.J. 1990. Hippocampus, amygdala, and memory deficits in rats. Behav. Brain Res. 37: 57-79.

Tassoni, G., Bucherelli, C., and Bures, J. 1992. Lateralized contributions of the cerebral cortex, parabrachial nucleus, and amygdala to acquisition and retrieval of passive avoidance reaction in rats: A functional ablation study. Behav. Neurosci. 106: 933-939.

Tassoni, G., Lorenzini, C.A., Baldi, E., Sacchetti, B., and Bucherelli, C. 1999. A peculiar pattern of temporal involvement of rat perirhinal cortex in memory processing. Behav. Neurosci. 113: 1161-1169. - 2000. Role of the perirhinal cortex in rats' conditioned taste aversion response memorization. Behav. Neurosci. 114: 875-881.

Treit, D. and Menard, J. 1997. Dissociations among the anxiolytic effects of septal, hippocampal, and amygdaloid lesions. Behav. Neurosci. 111: 653-658.

Treit, D., Pesold, C., and Rotzinger, S. 1993. Dissociating the anti-fear effects of septal and amygdaloid lesions using two pharmacologically validated models of rat anxiety. Behav. Neurosci. 107: 770-785.

Vanderwolf, C.H., Kelly, M.E., Kraemer, P., and Streather, A. 1988. Are emotion and motivation localized in the limbic system and nucleus accumbens? Behav. Neurosci. 27: 45-58.

Vazdarjanova, A. and McGaugh, J.L. 1998. Basolateral amygdala is not critical for cognitive memory of contextual fear conditioning. Proc. Natl. Acad. Sci. 95: 15003-15007.

. 1999. Basolateral amygdala is involved in modulating consolidation of memory for classical fear conditioning. J. Neurosci.
19: 6615-6622.

Vazdarjanova, A., Cahill, L., and McGaugh, J.L. 2001. Disrupting basolateral amygdala function impairs unconditioned freezing and avoidance in rats. Eur. J. Neurosci. 14: 709-718.

Wilensky, A.E., Schafe, G.E., and LeDoux, J.E. 1999. Functional inactivation of the amygdala before but not after auditory fear conditioning prevents memory formation. J. Neurosci. 19: 1-5.

. 2000. The amygdala modulates memory consolidation of fear-motivated inhibitory avoidance learning but not classical fear conditioning. J. Neurosci. 20: 7059-7066.

Williams, D.A., Frame, K.A., and LoLordo, V.M. 1991. Reexamination of contextual conditioning with massed versus distributed unconditioned stimuli. J. Exp. Psychol. Anim. Behav. Process. 17: 202-209.

Yin, H., Barnet, R.C., and Miller, R.R. 1994. Trial spacing and trial distribution effects in Pavlovian conditioning: Contributions of a comparator mechanism. J. Exp. Psychol. Anim. Behav. Process. 20: $123-134$.

Zhuravin, I.A. and Bures, J. 1991. Extent of the tetrodotoxin induced blockade examined by papillary paralysis elicited by intracerebral injection of the drug. Exp. Brain Res. 83: 687-690.

Received July 23, 2002; accepted in revised form April 15, 2003. 


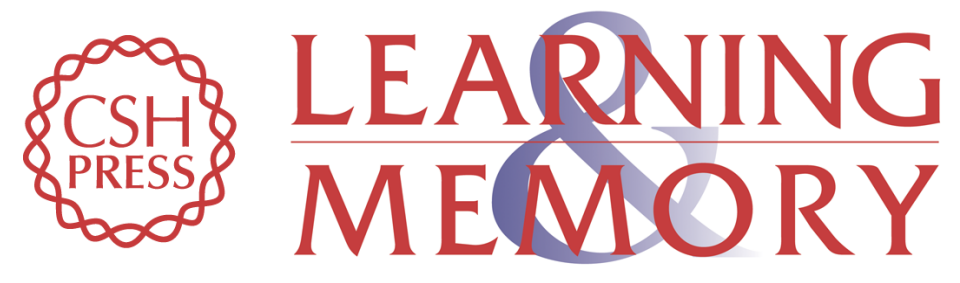

\section{Spared Anterograde Memory for Shock-Probe Fear Conditioning After Inactivation of the Amygdala}

Hugo Lehmann, Dallas Treit and Marise B. Parent

Learn. Mem. 2003, 10:

Access the most recent version at doi:10.1101//m.54103

References This article cites 68 articles, 8 of which can be accessed free at:

http://learnmem.cshlp.org/content/10/4/261.full.html\#ref-list-1

License

Email Alerting Receive free email alerts when new articles cite this article - sign up in the box at the Service top right corner of the article or click here. 